\title{
Tissue inhibitor of metalloproteinase 2 (TIMP-2) expression in adenocarcinoma pleural effusions
}

\author{
ENRICO GIARNIERI ${ }^{1}$, MAURO ALDERISIO ${ }^{1}$, RITA MANCINI ${ }^{1}$, CARLO FALASCA $^{1}$, \\ ALBERTO RICCI $^{2}$, SALVATORE MARIOTTA ${ }^{2}$ and MARIA ROSARIA GIOVAGNOLI ${ }^{1}$ \\ ${ }^{1}$ Cytopathology, and ${ }^{2}$ Department of Cardiovascular and Respiratory Sciences, II Faculty of Medicine, \\ University of Rome 'La Sapienza', St. Andrea Hospital, Rome 00189, Italy
}

Received July 17, 2007; Accepted October 22, 2007

\begin{abstract}
Serous effusions are frequently a clinical manifestation of metastatic disease, with lung, breast and ovarian carcinoma and mesothelioma leading the list. The diagnosis of malignant effusion signifies disease progression and is associated with a worsening patient prognosis. The ability to grow in a dense exudative fluid suggests that the malignant cells are capable of acquiring nutrients, surviving and proliferating, despite the lack of a solid-phase scaffold. During proliferation, neoplastic cells release ligands and matrix metalloproteinases (MMPs) into their environment, which dissolve the extracellular matrix (ECM). Tissue inhibitors of metalloproteinase (TIMPs) are endogenous regulators of MMPs, the principal enzymes responsible for the degradation of ECM in metastasis, and reduce their proteolytic activity. TIMP-2 has demonstrated an association between high tumor tissue expression levels and poor prognosis. The purpose of this preliminary study is to investigate, by immunocytochemistry, TIMP-2 expression in non-neoplastic and metastatic adenocarcinoma pleural effusions. We selected 16 cases of reactive mesothelio, 7 of normal mesothelio, 14 of lung adenocarcinoma, 9 from the ovary, 4 from the gastrointestinal tract and 3 from the breast. In 23/30 cases (76\%), we detected adenocarcinoma cells with strong TIMP- 2 expression. Positive TIMP-2 expression was found in $2 / 7$ cases $(28 \%)$ of normal and $2 / 16(12 \%)$ of reactive mesothelio. A statistical association was detected between TIMP-2 expression and metastatic adenocarcinoma cells compared to reactive and normal mesothelial cells $(\mathrm{p}<0.00003)$. The calculated sensitivities for TIMP-2 compared to CEA and Ber-EP4 were, respectively, $76.7,80.0$ and $93.3 \%$, and the specificities 82.6, 95.7 and $87.0 \%$. In conclusion, immunocytochemical detection of
\end{abstract}

Correspondence to: Dr Enrico Giarnieri, Cytopathology, II Faculty of Medicine, University of Rome 'La Sapienza', St. Andrea Hospital, Via di Grottarossa 1035, Rome 00189, Italy

E-mail: enrico.giarnieri@uniroma1.it

Key words: metalloproteinase 2, pleural effusions, metastasis, adenocarcinoma
TIMP-2 could be considered an interesting marker in metastatic adenocarcinoma pleural effusions, and could possibly be used as a component of an antibody panel in diagnostic cytopathology.

\section{Introduction}

Approximately half of all patients with metastatic cancer develop malignant pleural effusions. Because the patients are already terminally III, these effusions can present significant diagnostic and therapeutic challenges. In virtually all cases, symptoms are either present at the time of diagnosis or develop subsequently. Diagnosis is based on chest radiography followed by thoracentesis or thoracoscopy. Most malignant effusions are exudative; about one third are bloody. Cytology is positive for cancer cells in the initial pleural fluid specimens of $60 \%$ of those patients ultimately shown to have malignant effusions. The remaining $40 \%$ require repeat thoracentesis, pleural biopsy, thoracoscopy or other procedures to prove the presence of cancer through a molecular diagnostic approach. This supports the need for the development of new markers. Pleural fluid cytology is among the tools which offer the highest yield in diagnosing malignancy. The sensitivity of the test ranges from 40 to $87 \%$, depending mainly on the cytologist's training, the extent of pleural involvement, tumor type (yield is higher in adenocarcinoma) and markers of differentiation by immunocytochemistry. When pleural effusion is diagnosed as 'reactive', it is populated by mesothelial cells, morphologically different from their quiescent counterparts, which display activation, enlargement, proliferative activity and higher nuclear-to-cytoplasm ratios. In such cases, the expression levels of tumor markers in pleural effusions may help to establish the diagnosis of pleural malignancy by supporting a differential diagnosis between reactive mesothelial and neoplastic cells.

The microenvironment of neoplastic cells can define metastatic and growth potential. As neoplastic cells grow, they release ligands and matrix metalloproteinases (MMPs) into their environment, which dissolve the extracellular matrix (ECM). In response, the ECM itself releases soluble factors that enhance tumor cell invasion. When neoplastic cells reach the effusion they find a new environment, with nutrients and secreted growth factors, which can lead to the development of greater malignancy. In normal development, the regulation of 
ECM turnover is the result of a balance between tissue inhibitors of metalloproteinases (TIMPs) and MMPs; in cancer development, this regulation is lost (1-3).

The family of TIMPs consists of four distinct members, TIMP-1, -2, -3 and -4, which form stechiometric complexes with matrix metalloproteinases. The TIMP-1, $-2,-3$ and -4 family genes are localized on chromosomes Xp11-p11.4, 17q25, 22q12.1-13.2 and 3p25, respectively. The physiological activity of the TIMPs was studied by observing that TIMP-1, -3 and -4 expression is inducible and often tissue-specific, while TIMP-2 expression is constitutive and extensively expressed throughout the body (4-8). Deregulation of the MMP-TIMP balance can result in the progression of many human diseases, including cancer (9-11). In many human cancers, it has frequently been observed that TIMP upregulation is associated with a negative prognosis in metastatic breast cancer, colorectal cancer, lymphoma and non-small cell lung carcinoma (12-15).

The purpose of this preliminary study is to investigate, by means of immunocytochemistry, a TIMP-2 protein expression inhibitor, and whether expression could be useful in the selection of non-neoplastic and neoplastic adenocarcinoma cells in pleural effusions.

\section{Materials and methods}

Case selection. In this retrospective study, all serous pleural fluids were processed at our institute over a 1-year period and were retrieved from a cytopathology database. We randomly selected 16 cases of benign fluid containing reactive mesothelial cells, and 7 with normal morphological nonreactive mesothelial cells. The majority of the benign pleural effusions included in this study were selected in order to ensure that the benign mesothelial cells were abundant and well represented. We randomly selected 33 cases of malignant pleural fluid representing tumors that are commonly encountered in pleural fluid specimens. 14 cases were selected from pleural metastatic adenocarcinoma of the lung, 9 from the ovary, 4 from the gastrointestinal tract and 3 from the breast.

All specimens were received as fresh effusion, usually within 1 day following collection, with volumes of 40$1,500 \mathrm{ml}$. After being centrifuged for $5 \mathrm{~min}$ at $1,500 \mathrm{rpm}$, the supernatant was decanted and the sediment resuspended in $30 \mathrm{ml}$ of CytoLyte Solution (Cytyc Corp., Massachusetts, USA). The specimen was then recentrifuged for $10 \mathrm{~min}$ at $1,500 \mathrm{rpm}$ and the sediment resuspended in $20 \mathrm{ml}$ of PreservCyt solution (Cytyc Corp.). A monolayer slide was made using the ThinPrep 2000 Processor (Cytyc Corp.). From each specimen, 2-4 slides were prepared. Of these, 1 or 2 were stained using the Papanicolaou method, and viewed independently to confirm the diagnosis and presence of cells suitable for evaluation. Tumor type was determined according to clinical history, supported by surgical pathology diagnosis.

Immunocytochemical staining. Slides previously prepared by the ThinPrep procedure were rehydrated, and endogenous peroxidase blocked with $\mathrm{H}_{2} \mathrm{O}_{2}$ in methanol. Samples were then heated in citrate buffer (pressure cooker, $\mathrm{pH}$ 6.0, $5 \mathrm{~min}$ ) and washed in PBS. Blocking reagent followed by primary antibody and then biotinylated anti-mouse secondary antibody were added. The primary antibody used was an anti-TIMP-2 mouse monoclonal clone 3A4 (Zymed, USA).

After a further step of washing in PBS, slides were treated with avidin-biotin complex for $30 \mathrm{~min}$. Staining was performed with 3,3'-diaminobenzidin. and counterstaining with HE. At least 100 cells per view were counted. Cases were considered positive if at least $10 \%$ of cells were strongly stained for the antibody. When the primary origin of cancer cells was uncertain, immunocytochemistry staining, using monoclonal anti-CEA and anti-B72.3 (Zymed), was performed. The primary antibodies were omitted and replaced with preimmune serum in the negative control. Positive controls were adopted based on the manufacturer's instructions.

Statistical analysis. Statistical analysis was performed using the SPSS $^{\circledR}$ and Epi Info 6 software packages (SPSS for Windows, version 11.5 ; Epi Info 6, version 6.04b). Frequency tables were analyzed using the $\chi^{2}$ test. To assess the significance of the correlation between the categorical variables, Pearson correlation and/or Fisher's exact test were used.

TIMP-2 immunocytochemical staining was used as a dichotomous categorical variable (negative and positive) in the statistical analysis.

The traditional accuracy indicators, i.e. sensitivity and specificity, were computed using conventional formulas. Diagnoses were considered 'true positives' if they were in agreement with the consensus cytological diagnosis.

The cytological diagnosis of adenocarcinoma, reactive mesothelial cells and normal mesothelial cells were assigned to two categories, normal/reactive or adenocarcinoma.

\section{Results}

The immunocytochemical results are summarized in Tables I and II. In the effusions, TIMP-2 positive cells were easily detected, even when they were present in only small numbers. Of the cases identified as negative, effusions we detected in $2 / 7$ cases of non-reactive mesothelio (28\%) and 2/16 cases of reactive mesothelio (12\%) showing positive TIMP-2 immunoreaction staining. In contrast, strong staining was detected in 23/30 cases of effusion from metastatic adenocarcinoma (76\%) (Fig. 1C-E). TIMP-2 was expressed in 7/9 cases from the ovary (77\%), 12/14 of lung adenocarcinoma (85\%), 3/4 from the gastrointestinal tract $(75 \%)$ and $1 / 3$ from the breast $(33 \%)$. The characteristic staining pattern was cytoplasmatic, dense and strong, sometimes with accentuation at cell-cell borders. In some cases, nuclear and cytoplasmatic staining were detected contemporaneously. Histiocytes and other inflammatory cells did not exhibit TIMP-2 expression. Fisher's exact test showed a statistical association between TIMP-2 expression and adenocarcinoma cells $(\mathrm{p}<0.00003)$. TIMP-2 sensitivity and specificity were detected as $76.7 \%(57.3-89.4 \% \mathrm{CI})$ and $82.6 \%$ (60.5-94.3\% CI), respectively. Adenocarcinoma cells displayed CEA in 24/30 cases (77.2\%; $<<0.00001)$ and Ber-Ep4 in 28/30 $(89.8 \% ; p<0.00001)$ cases. While reactive effusion cells were found to be positive for CEA in $1 / 16$ cases $(6.2 \%)$ and for Ber-Ep4 in 3/16 (18.7\%) cases, no positive cells were found in normal effusion. The calculated sensitivities for TIMP-2, CEA and Ber-EP4 were, respectively, 76.7, 80.0 and 93.3\%, and the specificities 82.6, 95.7 and $87.0 \%$. 
Table I. Comparison of TIMP-2, CEA and Ber-EP4 immunocytochemistry results from adenocarcinoma.

\begin{tabular}{lrrc}
\hline Cancer & TIMP-2(\%) & CEA (\%) & Ber-EP4 (\%) \\
\hline Breast & $1 / 3(33.3)$ & $2 / 3(66.6)$ & $2 / 3(66.6)$ \\
Gastrointestinal & $3 / 4(75.0)$ & $3 / 4(75.0)$ & $4 / 4(100)$ \\
Lung & $12 / 14(85.7)$ & $11 / 14(78.5)$ & $13 / 14(92.8)$ \\
Ovary & $7 / 9(77.7)$ & $8 / 9(88.8)$ & $9 / 9(100)$ \\
Total & $23 / 30(67.9)$ & $24 / 30(77.2)$ & $28 / 30(89.8)$ \\
\hline
\end{tabular}

Table II. Comparison of sensitivity and specificity of immunocytochemistry.

\begin{tabular}{lcc}
\hline $\begin{array}{l}\text { Marker } \\
\text { antibodies }\end{array}$ & $\begin{array}{c}\text { \% Sensitivity } \\
\text { (CI 95\%) }\end{array}$ & $\begin{array}{c}\% \text { Specificity } \\
(\text { CI 95\%) }\end{array}$ \\
\hline TIMP-2 & $76.7(57.3-89.4)$ & $82.6(60.5-94.3)$ \\
CEA & $80.0(60.9-91.6)$ & $95.7(76.0-99.8)$ \\
Ber-Ep4 & $93.3(76.5-98.8)$ & $87.0(65.3-96.6)$ \\
\hline
\end{tabular}
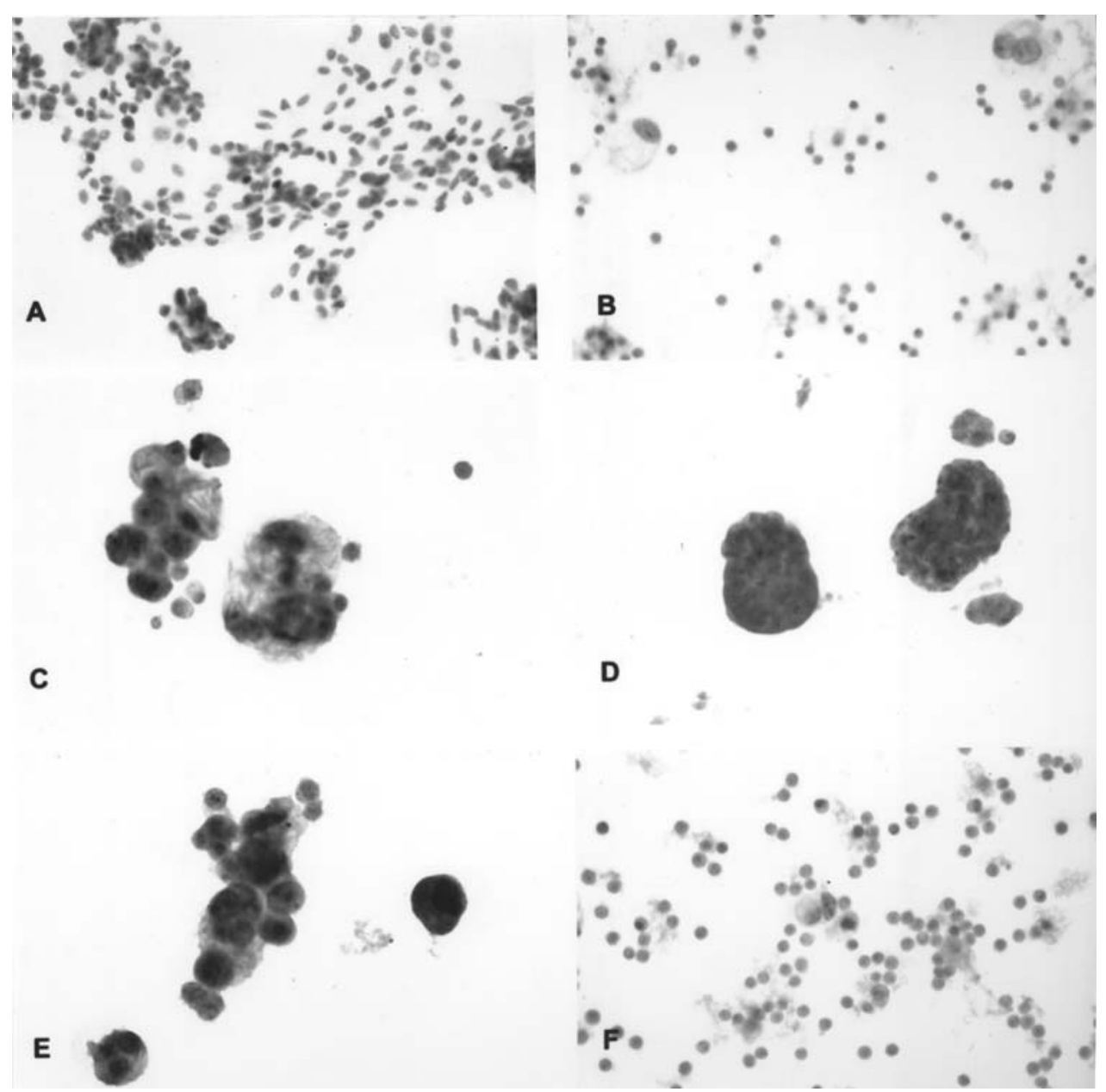

Figure 1. (A) Transudate pleural effusion. TIMP-2 reactivity in monolayer mesothelial cells is absent (magnification, x10). (B) Reactive pleural effusion. Note mesothelial cells with undetectable TIMP-2 cytoplasmatic immunoreaction (magnification, $x 10)$. (C) Neoplastic pleural effusion in patient with adenocarcinoma of the ovary. Aggregates of neoplastic cells with enlarged vacuolization show intense TIMP-2 immunoreactivity (magnification, x20). (D) Neoplastic pleural effusion in patient with breast adenocarcinoma. Clusters of neoplastic cells show TIMP-2 overexpression (magnification, x20). (E) Neoplastic pleural effusion in patient with lung adenocarcinoma. Note TIMP-2 cytoplasmatic overexpression (magnification, x20). (F) Reactive pleural effusion. Mesothelial cells show very low TIMP-2 cytoplasmatic immunoreaction (magnification, x10).

\section{Discussion}

The morphologic differentiation of reactive mesothelial versus adenocarcinoma cells in effusions can be a common daily challenge in a cytodiagnostic laboratory. During the course of the routine, false negatives are a common serious problem due to the misinterpretation of adenocarcinoma cells as reactive mesothelial cells. Conversely, an over-interpretation of reactive mesothelial cells as neoplastic cells can lead to false positive results $(16,17)$. Mesothelial cells react to a variety of stimuli and injuries and undergo proliferation and cellular alterations, including cytoplasmic and nuclear changes, that mimic malignant cytomorphology. These morphologic changes can be pronounced following radiation or chemotherapy, which are often associated with the surgical treatment of various malignancies. In view of the clinical implications 
of the presence of malignant cells, an accurate diagnosis becomes essential. Immunocytochemistry can be of great help in resolving such diagnostic difficulties. Unfortunately, the commercial antibodies available for a differential diagnosis of effusion show variations in sensitivity and specificity, and are subject to controversy concerning the markers or combination of markers adopted. Fixation and antigen retrieval methods and the types of materials (smears, cell block, thin prep) and different antibody dilutions used are but some of the sources of problems confronted in technical procedure standardization.

The present preliminary study shows that TIMP-2 overexpression is frequently detected in pleural effusions from adenocarcinoma, but not in non-neoplastic pleural effusion. Indeed, low or undetectable immunoreactivity was found prevalently in exudative and transudate effusions, confirming data previously reported by Eckeberg et al (18). A possible scenario, which explains the role of elevated TIMP-2 in advanced cancer, is that increased expression of TIMP-2 could be related to the increase of MMP expression during tumor progression. The relationship between TIMP-2 and MMPs has been described previously, and many studies support this hypothesis (19-21). Under normal conditions, MMP expression is finely controlled at multiple stages, including the regulation of transcription, translation and protein stability. MMPs are produced in a Zymogen form. Their activation and activity is regulated by their endogenous inhibitors, namely TIMPs. Conversely, disruption of the MMP-TIMP balance can result in a number of pathogenic processes, including tumor invasion, metastasis, angiogenesis and wound healing $(22,23)$. The overexpression of TIMP-2 in neoplastic cells could explain the presence of disfunctional proteins, incapable of interaction with MMPs, which block its enzymatic activity. It could also be speculated that the elevated expression of TIMP-2 is a response to local tissue degradation during cancer invasiveness. Neoplastic pleural effusion represents an interesting model through which to study the metastatic process because, at an advanced stage of the disease, neoplastic cells drop into the pleura and thus acquire biologically aggressive behavior and release many different proteins. The major effort entails understanding the proteins, and their features, which are involved in this process.

To date, data regarding TIMP-2 expression in metastatic pleural effusions have been inconsistent and a comparative evaluation, using pre-existing research, has been difficult. Nevertheless, Yano et al demonstrated, in an experimental study on nude mice, that the production of malignant pleural effusions is associated with high levels of urokinase-type plasminogen activator and with low levels of TIMP-2 (24). Different data have been reported for primitive lung cancer. Thomas et al observed that TIMP-2 is more frequently overexpressed at advanced stages of lung adenocarcinoma than in squamous cell carcinoma (25) and Suzuki et al, in a previous study, detected a correlation between TIMP-2 immunoreactivity and disease stage, observing up to $88 \%$ of positive cases in stages II, III and IV (26). Finally, Kumaki et al reported that, in pulmonary adenocarcinoma, TIMP-2 stained significantly more strongly in invasive areas than in the lipidic region, concluding that the development of scar cancer is favored by the increased expression of TIMP-2 and by tumor cells as they grow invasively (20).
These data are in agreement with our preliminary results, in which we detected TIMP-2 immunoreactivity in $85.7 \%$ of adenocarcinoma pleural effusions in primitive non-small cell lung cancer. This suggests that abnormal TIMP-2 proteins are involved in the pleural dissemination process.

Discordant data are reported in the literature regarding TIMP-2 and metastasizing colorectal cancer. We found TIMP-2 overexpression in $75 \%$ of cases affected by colorectal cancer with metastasis in pleural effusions. Studies regarding TIMP-2 expression in adenocarcinoma of the colon-rectum show that tissue concentrations and the epithelial mRNA expression of TIMP-2 decrease significantly in primary colorectal cancer (UICC stages III and IV) but increase in metastases (27). Li et al observed that, with the progression of invasion depth, lymph node metastasis and Duke's tumor stage, the activity and expression levels of MMP-2 and TIMP-2 gradually increased (28). Conversely, Moran et al observed a significant decrease in TIMP-2 in tumor samples when compared to non-tumoral tissue (29).

Metastasis from breast or ovarian adenocarcinoma is the most common etiology of pleural effusion, due to the presence of malignant cells in the effusions of female patients. Twothirds of ovarian carcinoma patients disseminate tumor cells in the peritoneal cavity and pleural effusion is present in more than $50 \%$ of stage IV patients (30). Davidson et al observed that adenocarcinoma cells in effusions showed a significant up-regulation of MMP-2 expression compared with primary tumors, with a concomitant down-regulation of TIMP-2 (31), while Kim et al detected high expression of TIMP-2 prevalently in serous carcinoma, suggesting that TIMP-2 may function in favor of tumor growth. They concluded that TIMP-2, with MMP-2, MMP-9 and MT1-MMP, is a valid marker of poor survival in advanced-stage ovarian carcinoma (32). In breast cancer, Vizoso et al detected elevated immunohistochemical scores for TIMP-2, TIMP-1 and MMP-9, significantly associated with a high rate of distant metastasis (33), and Brummer et al reported elevated TIMP-1 and -2 transcript levels in invasive breast carcinomas correlated with histological grade (34). In breast and ovary pleural effusion metastasis, respectively, we found that 33 and $77 \%$ of adenocarcinoma cells were TIMP-2 positive. TIMP-2 seems to play a limited role in detecting breast cancer in effusion cytology while, in ovary, lung and gastrointestinal cancer cells, elevated pattern points to an increase in invasive potential.

In our study, TIMP-2 expression is also correlated with CEA and Ber-EP4, which are frequently used in the differential diagnosis of adenocarcinoma in pleural effusions. A high rate of statistical association is observed between TIMP-2 and adenocarcinoma cells, with a sensitivity and specificity comparable to that reported for CEA and Ber-EP4 in the literature (35).

In conclusion, these preliminary data indicate that TIMP-2 expression may be extremely useful in a differential approach to reactive versus adenocarcinoma cells, rather than in the diagnosis of adenocarcinoma cell origin. More extensive case studies will be necessary in order to understand the role of TIMP-2 in pleural effusion metastasis, and to consider it an adjunctive marker for discerning adenocarcinoma cell origin in the cytologic diagnosis of malignant effusions. 


\section{References}

1. Freije JM, Balbin M, Pendas AM, Sanchez LM, Puente XS and Lopez-Otin C: Matrix metalloproteinases and tumor progression. Adv Exp Med Biol 532: 91-107, 2003.

2. Stetler-Stevenson WG and Yu AE. Proteases in invasion: matrix metalloproteinases. Semin Cancer Biol 11: 143-152, 2001.

3. Stetler-Stevenson WG, Aznavoorian S and Lotta LA: Tumor cell interactions with the extracellular matrix during invasion and metastasis. Ann Rev Cell Biol 9: 541-573, 1993.

4. Gomez DE, Alonso DF, Yoshiji H and Thorgeirsson UP: Tissue inhibitors of metalloproteinases: structure, regulation and biological functions. Eur J Cell Biol 74: 111-122, 1997.

5. Spurr NK, Goodfellow PN and Docherty AJ: Chromosomal assignment of the gene encoding the human tissue inhibitor of metalloproteinases to Xp11.1-p11.4. Ann Hum Genet 51: 189-194, 1987.

6. De Clerck Y, Szpirer C, Aly MS, Cassiman JJ, Eeckhout Y and Rousseau G: The gene for tissue inhibitor of metalloproteinases-2 is localized on human chromosome arm $17 \mathrm{q} 25$. Genomics 14 : 782-784, 1992.

7. Apte SS, Mattei MG and Olsen BR: Cloning of the cDNA encoding human tissue inhibitor of metalloproteinases-3 (TIMP-3) and mapping of the TIMP3 gene to chromosome 22. Genomics 19: 86-90, 1994.

8. Olson TM, Hirohata S, Ye J, Leco K, Seldin MF and Apte SS: Cloning of the human tissue inhibitor of metalloproteinase-4 gene (TIMP4) and localization of the TIMP4 and Timp4 genes to human chromosome 3 p25 and mouse chromosome 6 , respectively. Genomics 51: 148-151, 1998 .

9. Hojilla CV, Mohammed FF and Khokha R: Matrix metalloproteinases and their tissue inhibitors direct cell fate during cancer development. Br J Cancer 89: 1817-1821, 2003.

10. Yu Q and Stamenkovic I: Localization of matrix metalloproteinase 9 to the cell surface provides a mechanism for CD44-mediated tumor invasion. Genes Dev 13: 35-48, 1999.

11. Kleiner DE and Stetler-Stevenson WG: Matrix metalloproteinases and metastasis. Cancer Chemother Pharmacol 43: 42-51, 1999.

12. Ree AH, Florenes VA, Berg JP, Maelandsmo GM, Nesland JM and Fodstad O: High levels of messenger RNAs for tissue inhibitors of metalloproteinases (TIMP-1 and TIMP-2) in primary breast carcinomas are associated with development of distant metastases. Clin Cancer Res 9: 1623-1628, 1997.

13. Zeng ZS, Cohen AM, Zhang ZF, Stetler-Stevenson W and Guillem JG: Elevated tissue inhibitor of metalloproteinase 1 RNA in colorectal cancer stroma correlates with lymph node and distant metastases. Clin Cancer Res 8: 899-906, 1995.

14. Fong KM, Kida Y, Zimmerman PV and Smith PJ: TIMP1 and adverse prognosis in non-small cell lung cancer. Clin Cancer Res 8: $1369-1372,1996$

15. Kossakowska AE, Urbanski SJ and Edwards DR: Tissue inhibitor of metalloproteinases-1 (TIMP-1) RNA is expressed at elevated levels in malignant non-Hodgkin's lymphomas. Blood 11: 2475-2481, 1991.

16. Jacques SM and Selvaggi SM: Multiple peritoneal cytologies collected during laparotomy for gynecologic malignancy. Diagn Cytopathol 7: 482-486, 1991.

17. Hansen RM, Caya JG, Clowry LJ and Anderson T: Benign mesothelial proliferation with effusion. Clinicopathologic entity that may mimic malignancy. Am J Med 77: 887-892, 1984.

18. Eickelberg O, Sommerfeld CO, Wyser C, Tamm M, Reichenberger F, Bardin PG, Soler M, Roth M and Perruchoud AP: MMP and TIMP expression pattern in pleural effusions of different origins. Am J Respir Crit Care Med 156: 1987-1992, 1997.

19. Ruokolainen H, Paakko P and Turpeenniemi-Hujanen T: Tissue and circulating immunoreactive protein for MMP-2 and TIMP-2 in head and neck squamous cell carcinoma - tissue immunoreactivity predicts aggressive clinical course. Mod Pathol 19: 208-217, 2006.
20. Kumaki F, Matsui K, Kawai T, Ozeki Y, Yu ZX, Ferrans VJ and Travis WD: Expression of matrix metalloproteinases in invasive pulmonary adenocarcinoma with bronchioloalveolar component and atypical adenomatous hyperplasia. Am J Pathol 159: 2125-2135, 2001.

21. Fassina G, Ferrari N, Brigati C, Benelli R, Santi L, Noonan DM and Albini A: Tissue inhibitors of metalloproteases: regulation and biological activities. Clin Exp Metastasis 2000 18: 111-120.

22. Stetler-Stevenson WG: Matrix metalloproteinases in angiogenesis: a moving target for therapeutic intervention. J Clin Invest 13 1237-1241, 1999.

23. Seo DW, Li H, Guedez L, Wingfields PT, Diaz T, Salloum R, Wei BY and Stetler-Stevenson WG: TIMP-2 mediated inhibition of angiogenesis: an MMP-independent mechanism. Cell 114: 171-180, 2003

24. Yano S, Shinohara H, Herbst RS, Kuniyasu H, Bucana CD, Ellis LM and Fidler IJ: Production of experimental malignant pleural effusions is dependent on invasion of the pleura and expression of vascular endothelial growth factor/vascular permeability factor by human lung cancer cells. Am J Pathol 157: 1893-1903, 2000.

25. Thomas P, Khokha R, Shepherd FA, Feld R and Tsao MS Differential expression of matrix metalloproteinases and their inhibitors in non-small cell lung cancer. J Pathol 190: 150-156, 2000.

26. Suzuki M, Iizasa T, Fujisawa T, Baba M, Yamaguchi Y, Kimura H and Suzuki H: Expression of matrix metalloproteinases and tissue inhibitor of matrix metalloproteinases in non-small-cell lung cancer. Invasion Metastasis 18: 134-141, 1998.

27. Chan CC, Menges M, Orzechowski HD, Orendain N, Pistorius G, Feifel G, Zeitz $M$ and Stallmach A: Increased matrix metalloproteinase 2 concentration and transcript expression in advanced colorectal carcinomas. Int J Colorectal Dis 16: 133-140, 2001

28. Li BH, Zhao P, Liu SZ, Yu YM, Han M and Wen JK: Matrix metalloproteinase-2 and tissue inhibitor of metallo-proteinase-2 in colorectal carcinoma invasion and metastasis. World $\mathrm{J}$ Gastroenterol 28: 3046-3050, 2005.

29. Moran A, Iniesta P, Garcia-Aranda C, De Juan C, Diaz-Lopez A, Sanchez-Pernaute A, Torres AJ, Diaz-Rubio E, Balibrea JL and Benito M: Clinical relevance of MMP-9, MMP-2, TIMP-1 and TIMP-2 in colorectal cancer. Oncol Rep 13: 115-120, 2005.

30. Hoskins WJ, Perez CA and Young RC: Epithelial ovarian cancer. In: Principles and Practice of Gynecologic Oncology. 3rd edition. Ozols RF, Rubin SC, Thomas GM and Roboy SJ (eds). Lippincott Williams \& Wilkins, Philadelphia, pp981-999, 2000.

31. Davidson B, Reich R, Berner A, Givant-Horwitz V, Goldberg I, Risberg B, Kristensen GB, Trope CG, Bryne M, Kopolovic J and Nesland JM: Ovarian carcinoma cells in serous effusions show altered MMP-2 and TIMP-2 mRNA levels. Eur J Cancer 37: 2040-2049, 2001.

32. Kim TJ, Rho SB, Choi YL, Choi CH, Lee JW, Bae DS, Ahn G, Lee JH and Kim BG: High expression of tissue inhibitor of metalloproteinase-2 in serous ovarian carcinomas and the role of this expression in ovarian tumorigenesis. Hum Pathol 37: 906-913, 2006.

33. Vizoso FJ, Gonzàlez LO, Corte MD, Rodríguez JC, Vázquez J, Lamelas ML, Junquera S, Merino AM and García-Muñiz JL: Study of matrix metalloproteinases and their inhibitors in breast cancer. Br J Cancer 96: 903-911, 2007.

34. Brummer O, Athar S, Riethdorf L, Löning T and Herbst H: Matrixmetalloproteinases 1,2 , and 3 and their tissue inhibitors 1 and 2 in benign and malignant breast lesions: an in situ hybridization study. Virchows Arch 435: 566-573, 1999.

35. Dabbs DJ (ed): Diagnostic Immunohistochemistry. Churchill Livingstone, London, 2002. 